\title{
NORMAL SUBGROUPS OF FUCHSIAN GROUPS WITH FIXED PARABOLIC CLASS NUMBER
}

\author{
MARC BERGER ${ }^{1}$
}

\begin{abstract}
Let $F$ be a finitely generated Fuchsian group of the first kind. We provided necessary and sufficient conditions such that, for any $t \geqq 1, F$ has only a finite number of normal subgroups with $t$ parabolic classes.
\end{abstract}

1. Introduction. In this paper we consider finitely generated Fuchsian groups $F$ of the first kind. L. Greenberg [1] has shown that for any $t \geqq 1$ there are only a finite number of normal subgroups of the $(2,3, \infty)$ modular group with $t$ parabolic classes. M. Newman [3] has obtained the same result for any $(p, q, \infty)$ triangle group where $(p, q)=1$. We shall now resolve this problem for an arbitrary Fuchsian group by providing necessary and sufficient conditions for this to occur.

2. Preliminaries. Let $D$ denote the unit disc $\{z:|z|<1\}$. A Fuchsian group $F$ is a discrete subgroup of $L(D)$, the group of conformal homeomorphisms of $D$. A Fuchsian group $F$ is said to be of the first kind if the closure, of the set of fixed points of nonelliptic elements of $F$ distinct from the identity, is the entire boundary of $D$. tion:

Finitely generated Fuchsian groups of the first kind have the presenta-

Generators: $a_{1} b_{1}, \cdots, a_{g} b_{g}, e_{1}, \cdots, e_{k}, p_{1}, \cdots, p_{r}$

Defining relations: $e_{1}^{\nu_{1}}=e_{2}^{\nu_{2}}=\cdots=e_{k}^{\nu_{k}}=1$

$$
\left(\prod_{i=1}^{g} a_{i} b_{i} a_{i}^{-1} b_{i}^{-1}\right) e_{1}, \cdots, e_{k}, p_{1}, \cdots, p_{r}=1 .
$$

If $F$ has the above presentation, we say that $F$ has signature $\left(g ; v_{1}, \cdots\right.$, $\left.v_{k} ; r\right)$ and denote the group by $F\left(g ; v_{1}, \cdots, v_{k} ; r\right)$. The signature tells us there are precisely $k$ nonconjugate (in $F$ ) maximal cyclic subgroups of

Received by the editors April 12, 1972.

AMS (MOS) subject classifications (1970). Primary 20H10, 30A58; Secondary 32J15, $20 \mathrm{~F} 05$.

Key words and phrases. Discontinuous group, Riemann surface, parabolic class.

${ }^{1}$ These results are contained in the author's doctoral thesis which was written under the direction of Professor Leon Greenberg at the University of Maryland.

(c) American Mathematical Society 1973 
$F$ generated by an elliptic element of order $\nu_{i}, i=1, \cdots, k$, and $r$ nonconjugate (in $F$ ) maximal cyclic subgroups of $F$ generated by a parabolic element. $F$ is said to have $r$ parabolic classes. The letter $g$ denotes the genus of $F$.

The following theorem (Knopp and Newman [2]) will be useful.

THEOREM 1. Let $N$ be a normal subgroup of a Fuchsian group $F$ with finite index $\mu$. Suppose that the $r$ parabolic generators in $F$ have exponent $m_{i}$ modulo $N, 1 \leqq i \leqq r$ (that is $m_{i}$ is least positive integer such that $p_{i}^{m_{i}} \in N$ ). Then the number $t$ of parabolic classes of $N$ is given by $t=\mu \sum_{i=1}^{r}\left(1 / m_{i}\right)$.

Proof. Since $F$ acts discontinuously on $D$ we obtain quotient surfaces $D / N$ and $D / F . N$ being a normal subgroup of $F$ implies the (branched) covering $\phi: D / N \rightarrow D / F$ is normal. $D / F$ is a closed surface with $r$ punctures and $D / N$ is a closed surface with $\mu / m_{i}$ punctures over each of the $r$ punctures in $D / F$. The theorem now follows.

\section{Initial results.}

Lemma 2. Let $p$ and $q$ be distinct primes with the properties $q>p$ and $q \equiv 1(\bmod p)$. There exists a nonabelian group of order $p q$.

Proof. Let $H=\langle b\rangle, K=\langle a\rangle$ where $a^{p}=b^{q}=1$. Since $q \equiv 1(\bmod p)$ we can, by the Fermat theorem, choose an integer $r$ such that $r \not \equiv 1(\bmod q)$ but $r^{p} \equiv 1(\bmod q)$. Define $\psi\left(a^{i}\right) b=b^{r^{i}}, 1 \leqq i \leqq p$. Then $\psi\left(a^{i}\right) b$ is clearly an automorphism of $H$ and $\psi$ is a homomorphism from $K$ to the automorphism group of $H$. Therefore we can form the semidirect product $G$ of $H$ by $K$.

Since

$$
|G|=|H K|=(|H| \cdot|K|) /|H \cap K|=p q
$$

$G$ has order $p q$. Since $\psi(a) b=a^{-1} b a=b^{r}, r \not \equiv 1(\bmod q)$ we conclude that $G$ is nonabelian.

THEOREM 3. There exists an infinite number of groups $G$ of order pn, $p$ prime, $n$ a positive integer, with generators satisfying the relations

$$
x^{p}=y^{p}=(x y)^{n}=z^{n}=1 .
$$

Proof. Let $G$ be the nonabelian group of order $p q$ in Lemma 2. Since $q>p$ there is a unique $q$-Sylow subgroup generated by some element $z \in G$. Let $a$ generate one of the $p$-Sylow subgroups. Consider the element $y=a z$. Then $y$ cannot have order $q$. For, if it did, the uniqueness of our $q$-Sylow subgroup would imply $a z=z^{k}$ for some $1 \leqq k<p$ and hence $a=$ $z^{k-1}$. But $a$ has order $p$ and $z$ has order $q$, therefore $\langle a\rangle$ and $\langle z\rangle$ can only have the identity element in common. Hence $y$ must have order $p(y$ cannot have order $p q$ since $G$ is noncyclic). Setting $x=a^{-1}$ we obtain the 
relations $x^{p}=y^{p}=(x y)^{q}=1$. Clearly $x$ and $y$ generate $G$. By the Dirichlet theorem on primes there are an infinite number of primes $q$ satisfying $q \equiv 1(\bmod p)$, and the theorem follows.

We shall denote the groups of the previous theorem by $[p, p, n]$.

Let $G$ be a group with an abelian subgroup $A$ of finite index $n$. Let $X=\left\{x_{1}, x_{2}, \cdots, x_{n}\right\}$ be a set of coset representatives of the right cosets $A g$. If $g \in G$ we define a mapping $\tau$ of $G$ into $A$ by

$$
\tau(g)=x_{1} g\left(\overline{x_{1} g}\right)^{-1} x_{2} g\left(\overline{x_{2} g}\right)^{-1} \cdots x_{n} g\left(\overline{x_{n} g}\right)^{-1}
$$

where $\bar{g} \in X$ is the representative of the right coset $A g$. The mapping $\tau$ is a homomorphism of $G$ into $A$ called the transfer of $G$ into $A$. If $A$ is contained in the center of $G, \tau(g)=g^{n}$.

\section{Main result.}

DEFINITION. A Fuchsian group $F$ is said to have the finite class property (FCP) if for any integer $t \geqq 1$ there are only a finite number of normal subgroups of $F$ with $t$ parabolic classes.

THEOREM 4. A Fuchsian group $F$ has $(F C P)$ if and only if (1) $F$ is of genus zero, (2) F has exactly one parabolic class and (3) the elliptic generators of $F$ have pairwise relatively prime orders. That is if and only if $F=$ $F\left(0 ; v_{1}, v_{2}, \cdots, v_{k} ; 1\right),\left(v_{i}, v_{j}\right)=1, i \neq j, i, j=1, \cdots, k$.

Proof. We shall prove the necessity first. Our method is to show that if $F$ is not of the above type we can construct for some $t \geqq 1$ an infinite number of normal subgroups with $t$ parabolic classes.

Case 1. We first show that $F$ cannot have more than one parabolic class.

Suppose $F$ has two or more parabolic classes $p_{1}, p_{2}, \cdots, p_{r}, p$ with relation:

$$
\left(\prod_{i=1}^{g} a_{i} b_{i} a_{i}^{-1} b_{i}^{-1}\right) e_{1}, \cdots, e_{k}, p_{1}, \cdots, p_{r}=p .
$$

Denote by $C_{q}$ the cyclic group of prime order $q$ generated by $x$. Define the homomorphism $\phi: F \rightarrow C_{q}$ by

$$
\begin{aligned}
& \phi\left(e_{j}\right)=\phi\left(a_{i}\right)=\phi\left(b_{i}\right)=1, \quad 1 \leqq j \leqq k, 1 \leqq i \leqq g, \\
& \phi\left(p_{1}\right)=x^{m_{1}}, \cdots, \phi\left(p_{r}\right)=x^{m_{r}}, \phi(p)=x,
\end{aligned}
$$

where $1 \leqq m_{i}<q, \sum_{i=1}^{r} m_{i} \equiv 1(\bmod q)$.

Since $q$ is prime the order of $x^{m_{i}}$ is $q, i=1, \cdots, r$. Let $N=\operatorname{ker} \phi$. Then $p_{i}, p, i=1, \cdots, r$, have exponent $q$ modulo $N$. By Theorem $1, N$ has $r+1$ parabolic classes. Letting $q \rightarrow \infty$ we have obtained an infinite number of normal subgroups $N$ with $t=r+1$ parabolic classes. 
Case 2. Suppose $F$ has positive genus $g$. Consider the homomorphism $\phi: F \rightarrow[2,2, n]$ defined by

$$
\begin{aligned}
& \phi\left(a_{1}\right)=x, \quad \phi\left(b_{1}\right)=z, \quad \phi(p)=z^{-2} \text { and } \\
& \phi\left(a_{j}\right)=\phi\left(b_{j}\right)=\phi\left(e_{i}\right)=1, \quad 1 \leqq i \leqq k, 2 \leqq j \leqq g .
\end{aligned}
$$

This is in fact a homomorphism, for in $[2,2, n] \simeq D_{n}$, the dihedral group on $n$ letters, $x z x^{-1}=z^{-1}$ or $x z x^{-1} z^{-1}=z^{-2}$.

Let $n=2 m$ and set $N=\operatorname{ker} \phi$. Then $\phi\left(p^{m}\right)=z^{-2 m}=z^{-n}=1$ implies $p^{m} \in N$. Clearly $\mu=|F| N \mid=2 \cdot 2 m=4 m$. Therefore setting $t=4$ and letting $m \rightarrow \infty$ we have found an infinite number of normal subgroups with $t=4$ parabolic classes.

Case 3. If $F$ has genus zero and only one parabolic class generator $p$, $F$ must have at least two elliptic elements $e_{1}, e_{2}$. This is an immediate consequence of the relation $(*)$. We shall now assume that there is a prime $q$ dividing the orders $\nu_{1}$ and $\nu_{2}$ of $e_{1}$ and $e_{2}$ respectively. Define the homomorphism $\phi: F \rightarrow[q, q, n]$ by

$$
\phi\left(e_{1}\right)=x, \quad \phi\left(e_{2}\right)=y, \quad \phi(p)=x y \quad \text { and } \quad \phi\left(e_{j}\right)=1, \quad 3 \leqq j \leqq k .
$$

Setting $N=\operatorname{ker} \phi$ we have $p^{n} \in N$ and $|F / N|=q n$. Taking $t=q$ and letting $n \rightarrow \infty$ we obtain an infinite number of normal subgroups $N$ with $t=q$ parabolic classes.

These are clearly the only cases and the necessity is completed.

To prove the sufficiency, the following lemma is used.

LEMMA 5. Let F be a Fuchsian group with signature

$$
\sigma=\left(0 ; v_{1}, \nu_{2}, \cdots, v_{k} ; 1\right)
$$

where $\left(v_{i}, v_{j}\right)=1, i \neq j, i, j=1,2, \cdots, k$. Let $N$ be a normal subgroup of finite index in $F$ with $t$ parabolic generators. Let $G=F / N$. $G$ has a subgroup $U$ such that

(a) $U \subset$ center of $G$,

(b) $[G: U] \leqq t^{t}$.

Proof. Let $\left\{x_{i}\right\}_{i=1}^{k}, z$ be the images of the elliptic and parabolic generators in $G$. Let $U=\bigcap_{g_{\in} G} g\langle z\rangle g^{-1}$. $U$ is clearly a normal subgroup of $G$. For $g \in G$ let $\alpha_{g}: U \rightarrow U$ be the automorphism $(u) \alpha_{g}=g^{-1} u g$. We have the relations

$$
\begin{array}{r}
\alpha_{x_{1}} \nu_{1}=\alpha_{x_{2}} v_{2}=\cdots=\alpha_{x_{k}} v_{k}=1, \\
\alpha_{x_{1}} \alpha_{x_{2}} \cdots \alpha_{x_{k}}=\alpha_{z}=1 .
\end{array}
$$


Since $U$ is cyclic its automorphism group is abelian and the $\alpha_{x_{i}}, i=1, \cdots$, $k$, commute. Solving equation (†) for $\alpha_{x_{j}}$ in terms of the remaining $\alpha_{x_{i}}$, $i \neq j, i=1, \cdots, k$, we conclude that the order of $\alpha_{x_{j}}$ divides $v_{1} \nu_{2} \cdots$ $v_{j-1} v_{j+1} \cdots v_{k}$. But the order of $\alpha_{x_{j}}$ divides $v_{j}$ and $\left(v_{i}, v_{j}\right)=1, i \neq j, i=1$, $\cdots, k$, and therefore $\alpha_{x_{j}}$ has order 1 , that is $\alpha_{x_{j}}=1$. This procedure works for all $\alpha_{x_{j}}, j=1, \cdots, k$, hence $U$ is central in $G$. Let $|G|=\mu$ and $|\langle z\rangle|=n$. By Theorem 1,

$$
[G:\langle z\rangle]=\left[G: g\langle z\rangle g^{-1}\right]=t,
$$

and there are $[G: N\langle z\rangle]$ subgroups conjugate to $\langle z\rangle$ with

$$
[G: N\langle z\rangle] \leqq[G:\langle z\rangle] \leqq t,
$$

we conclude that $[G: U] \leqq t^{t}$

We now return to the proof of our theorem. Let us consider the following homomorphism of $F$ into $U$ :

$$
F \stackrel{\phi}{\longrightarrow} F / N \stackrel{\tau}{\longrightarrow} U
$$

The mapping $\phi$ is the canonical homomorphism and $\tau$ is the transfer homomorphism. Let $H=G|U, G=F / N, r=| U|, s=| H \mid$. Then $s=\mu / r$ and by the lemma $s \leqq t^{t}$. Let $U^{s}$ denote the subgroup of $U$ consisting of all $s$ th powers.

Since $U \subset Z(G)$, for all $g \in G, \tau(g)=g^{s}$. Hence $\tau \circ \phi$ is an epimorphism of $F$ onto $G^{s} \supset U^{s}, G^{s} \subset U$. Since an abelian homomorphic image of $F$ has order at most $v_{1} v_{2} \cdots v_{k}$, we obtain $\left|U^{s}\right| \leqq\left|G^{s}\right| \leqq v_{1} \nu_{2} \cdots v_{k}$. As $U$ is cyclic we get $r / s \leqq r /(r, s)=\left|U^{s}\right|$. Therefore $r \leqq s\left|U^{s}\right|$ or $r \leqq v_{1} v_{2} \cdots v_{k} t^{t}$.

Since $\mu=r s$ we conclude $\mu \leqq v_{1} v_{2} \cdots v_{k} t^{2 t}$.

Since the number of subgroups of bounded index in a finitely generated group is finite (Hall [4]) we obtain our result.

\section{REFERENCES}

1. L. Greenberg, Note on normal subgroups of the modular group, Proc. Amer. Math. Soc. 17 (1966), 1195-1198. MR 33 \#7423.

2. M. I. Knopp and M. Newman, Congruence subgroups of positive genus of the modular group, Illinois J. Math. 9 (1965), 577-583. MR 31 \#5902.

3. M. Newman, Two-generator groups and parabolic class numbers, Proc. Amer. Math. Soc. 31 (1972), 51-53.

4. M. Hall, Jr., The theory of groups, Macmillan, New York, 1959, MR 21 \#1996.

Department of Mathematics, Millersville State College, Millersville, PenNSYlvania 17551

Current address: Division of Mathematics, District of Columbia Teachers College, Washington, D.C. 20009 